\title{
The spectroscopic observations of CoRoT asteroseismic targets with HARPS
}

\author{
Poretti, E., Rainer, M., Mantegazza, L., Floquet, M., Mathias, Ph., Amado, P., \\ Aerts, C., Uytterhoeven, K. and the CoRoT Seismology Ground-Based Working \\ Group
}

\begin{abstract}
CoRoT photometric measurements of asteroseismic targets need complementary ground-based spectroscopic observations. We are using the planet-hunter HARPS spectrograph attached to the $3.6 \mathrm{~m}$-ESO telescope in the framework of two consecutive Large Programmes. We discuss its use to study line-profile variations and we report on a specific result obtained for the $\delta$ Sct star HD 170699.
\end{abstract}

\section{Introduction}

Ground-based spectroscopic observations began at once with the launch of the CoRoT satellite. They involve several high-resolution échelle spectrographs: FEROS and HARPS at ESO-La Silla (Chile), FOCES at Calar Alto (Spain), SOPHIE at the Observatoire Haute Provence (France), FIES at NOT and HERMES at Mercator [6] (both at the Observatorio Roque de los Muchachos, La Palma, Spain). A small number of spectra were also obtained with CORALIE at ESO-La Silla and HERCULES at Mt. John Observatory (New Zealand). After the completion of the ESO Large

Poretti Ennio, Rainer Monica, Mantegazza Luciano

INAF-OA Brera, Via E. Bianchi 46, 23879 Merate, Italy.

Floquet Michele

GEPI, Observatoire de Paris, 5 place Jules Janssen, 92195 Meudon Cedex, France

Mathias Philippe

IRAP, 57 Avenue d'Azereix, 65008 Tarbes, France

Amado Pedro

Instituto de Astrofísica de Andalucía, Apartado 3004, 18080 Granada, Spain

Aerts Conny

Instituut voor Sterrenkunde, KU Leuven, Celestijnenlaan 200D, 3001, Leuven, Belgium

Uytterhoeven Katrien

Instituto de Astrofísica de Canarias (IAC), Tenerife, Spain 
Programme with FEROS (LP 178-D.0361, 60 nights in total), we obtained two subsequent Large Programmes with HARPS, the famous planet-hunter échelle spectrograph primarily used for the measurements of radial velocities. The first Large Programme with HARPS (LP 182.D-0356) consisted of 45 nights of observations between December 2008 and December 2009, while the current LP 185.D-0056 started on June 2010 and will end in January 2013. As of now, we have taken about 2500 HARPS spectra of CoRoT asteroseimic targets. The high-resolution spectra make the detection of high-degree modes possible [4] since the stellar disk can be spatially resolved thanks to the Doppler shifts induced by the star's rotation. Therefore, by measuring the observed variations in the line profiles, it is possible to know what kind of modes are excited in the stars and to assign the spherical wavenumbers $(\ell, m)$ to each of them. The HARPS spectra are reduced and normalized in a homogeneous way using a semi-automated pipeline developed at INAF-OAB.

\section{HARPS: improvements in the instrument setup}

HARPS is able to operate in two configurations: high accuracy (HAM mode, with $\mathrm{R}=115,000$ ) or high efficiency (EGGS mode, with $\mathrm{R}=80,000$ ). At the telescope, the HARPS pipeline provides an estimate of the signal-to-noise ratio (SNR) at three different wavelengths (4500, 5000, and $6500 \AA$ ). We chose to work mostly in the EGGS configuration, aiming at a signal-to-noise ratio (SNR) estimated at the telescope of about 200. In the reduction process we computed the SNRs taking into account photon noise, readout noise, and flat field correction. We could infer that for our stars (B-A-F spectral types) the SNR values given by the HARPS pipeline at $5500 \AA$ are a little too optimistic when compared with the values we measured between 5802 and $5825 \AA$. The ratio between the two SNR values is around 1.23 for A-F stars and around 1.28 for B stars.

Analysing the HARPS data obtained during the first observing runs (14-24 December 2008 and 3-8 January 2009) of the Large Programme ESO LP 182.D-0356, we found the presence of a disturbing feature in the spectra, i.e., an oscillation with an amplitude of about $0.05 \%$ of the continuum. It was the first time that this problem was reported. In fact, our approach to the data is different than the one usually used with the HARPS spectra. Usually the spectra are used to compute radial velocities of cool stars, while we search for line profile variations in hot stars. In particular, this feature became evident only by averaging a large number of spectra of the same line (Fig. 1, upper red curve), while it is practically invisible in a single spectrum. During the observing run of June 2009, several attempts were made to understand the nature of this problem, which in the meantime had worsened. It was found that the filters in front of the calibration lamps were misaligned after a check-up in May 2009 , and this enhanced the amplitude of the oscillations, which reached a value of about $2 \%$ of the continuum. Realigning the filters decreased the feature's amplitude to the previous $0.05 \%$ of the continuum, but did not eliminate it. With the help of the ESO staff we were able to identify the source of this problem as an interfer- 
ence created by the flat-field filters (red and blue). In fact, the issue was solved by taking away the blue filter: the subsequent flat-field correction was free from the instrumental effect (Fig. 1, lower black curve).

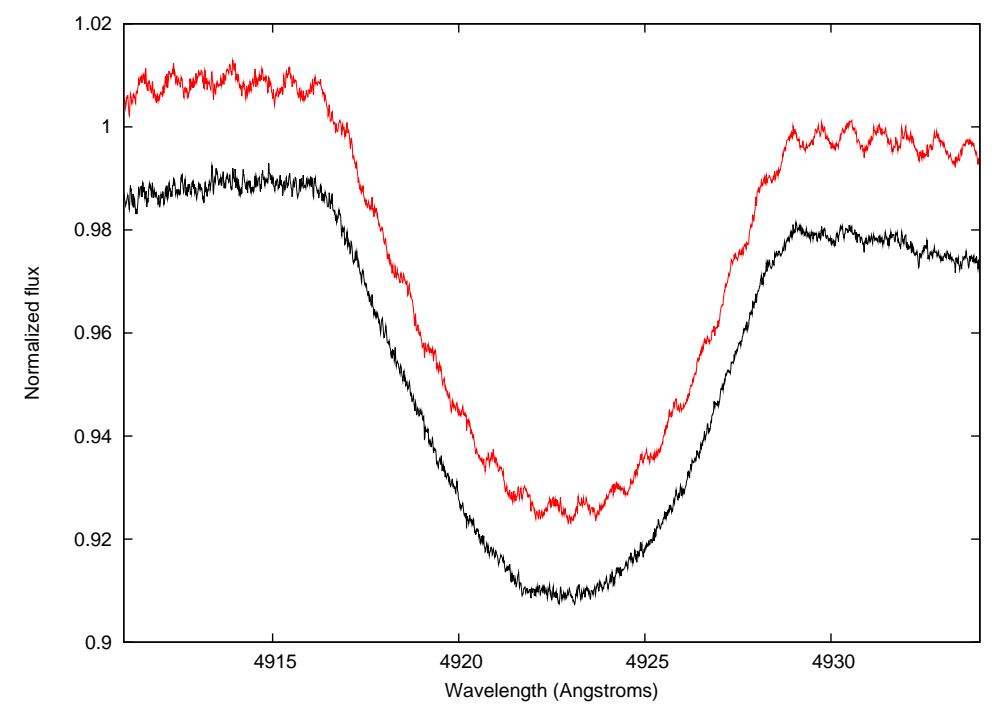

Fig. 1 Average $4921 \AA$ A He I line of the Be star HD 51452: the instrumental effect (upper red curve) was eliminated by removing the blue filter located on the optical path of the HARPS calibration lamp (lower black curve).

\section{Evidence of high-degree modes excited in $\delta$ Sct stars}

Several papers were published using the spectra observed in the ground-based programme (see the special A\&A vol. 506) and a lot of work is still in progress. The data have been used in several ways, ranging from frequency analysis to the identification of the physical parameters of the stars. We present here the case of the $\delta$ Sct stars, which are main-sequence or slightly evolved stars showing multiperiodic light curves. CoRoT introduced a dramatic change in terms of both number of identified frequencies and of the involved physics: hundreds of frequencies were detected in the light curve of HD 50844 [5]. The main goal of the spectroscopic observations is to determine if the observed frequencies can be associated with pulsation modes. The mode identification was performed by fitting the amplitude and phase variations of each mode across the average line profile of each HARPS spectrum [3], [8]. Figure 2 shows the phase diagram of the high-frequency term $f=48.26 \mathrm{c} / \mathrm{d}$ detected in the HARPS spectra of HD 170699. The total amount of the phase shifts suggests that it should be a $\ell=16 \pm 1$ mode [7]. This frequency is the second detected 
Poretti et al.

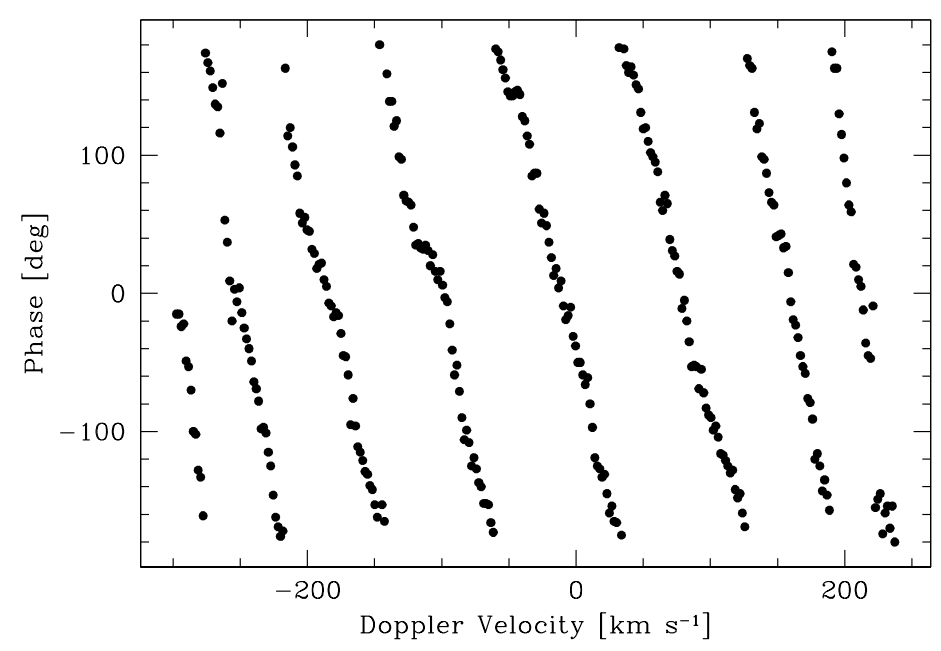

Fig. 2 HD 170699: phase variations across the line profile of the $f=48.26 \mathrm{~d}^{-1}$ term (HARPS spectra)

in the spectroscopic data and the $374^{\text {th }}$ in the CoRoT photometric light curve, with an amplitude of about $50 \mu \mathrm{mag}$. Therefore, the case of HD 170699 seems to support the conclusion made in the case of HD 50844 [5], i.e., that cancellation effects are not sufficient in completely removing the variations of the integrated flux associated with $\ell \geq 5$ modes. Two other physical mechanisms can be invoked to explain the rich frequency spectra observed in $\delta$ Sct stars: granulation effects [1] or island modes, e.g., [2]. These three different scenarios contribute to make the modeling of $\delta$ Sct stars a challenging task for theoreticians. In particular, the visibility of island modes may be particularly relevant in the case of HD 170699. We measured $v_{\mathrm{eq}} \sin i=270 \mathrm{~km} \mathrm{~s}^{-1}$, a value very close to the break-up velocity, and we can infer that HD 170699 is seen equator-on.

\section{References}

1. Kallinger, T., Matthews, J.M.: ApJ 711, L35 (2010)

2. Lignières, F., Georgeot, B.: A\&A 500, 1173 (2009)

3. Mantegazza, L.: Delta Scuti stars and related stars, M. Breger \& M.H. Montgomery Eds., ASP Conf. Series 210, 38 (2000)

4. Mantegazza, L., Poretti, E., Zerbi, F.M.: A\&A 366, 547 (2001)

5. Poretti, E., Michel, E., Garrido, R., et al.: A\&A 506, 85 (2009)

6. Raskin, G., van Winckel, H., Hensberge, H., et al.: A\&A 526, A69 (2011)

7. Telting, J.H., Schrijvers, C.: A\&A 317, 723 (1997)

8. Zima, W.: Communications in Asteroseismology 155, 17 (2008) 